\title{
Archéopages
}

Archéopages

Archéologie et société

\section{Habitats antiques en terre à Lons-le-Saunier}

Variété et évolution des techniques

Roman earthen buildings at Lons-le-Saunier. Diversity and development of techniques

Hábitats antiguos de barro en Lons-le-Saunier. Variedad y evolución de las técnicas

\section{Valérie Viscusi}

\section{(2) OpenEdition \\ Journals}

Édition électronique

URL : https://journals.openedition.org/archeopages/1265

DOI : 10.4000/archeopages.1265

ISSN : 2269-9872

Éditeur

INRAP - Institut national de recherches archéologiques préventives

Édition imprimée

Date de publication : 1 mars 2016

Pagination : 94-99

ISSN : 1622-8545

Référence électronique

Valérie Viscusi, « Habitats antiques en terre à Lons-le-Saunier », Archéopages [En ligne], 42 |

04-07/2015, mis en ligne le 01 juillet 2017, consulté le 03 juin 2021. URL : http://

journals.openedition.org/archeopages/1265; DOI : https://doi.org/10.4000/archeopages. 1265

(c) Inrap 


\section{Habitats antiques en terre à Lons-le-Saunier Variété et évolution des techniques}

Valérie Viscusi Inrap, UMR 6249 “Chrono-environnement»

\section{4}

1. Coordination V. Viscusi, Inrap et J.-L. Mordefroid, ville de Lons-le-Saunier. 2. Sous la direction de V. Viscusi Simonin, Inrap, rapport en cours.
Implantée au débouché d'une reculée entaillant le plateau jurassien, l'agglomération antique de Lons-le-Saunier (Jura) était probablement le cheflieu d'un pagus séquane. Si diverses découvertes anciennes, essentiellement au cours du XIX ${ }^{\mathrm{e}}$ siècle, ont permis de révéler la présence de vestiges antiques, leur importance a été mise en évidence par plusieurs opérations de fouille ou de surveillance de travaux menées à partir des années 1960 (Rothé, 2001) et par les nombreuses observations réalisées par l'équipe du service d'archéologie de la ville. Parmi celles-ci, l'importante fouille de la place de la Comédie dirigée par J.-L. Mordefroid en 1989-1990 a notamment permis la découverte d'un collecteur qui contenait un très riche mobilier (céramique, verre, métal, lampes à huile, intailles...) tout à fait exceptionnel (Mordefroid et al., 1990). La mise en évidence d'un niveau d'occupation laténien a permis de confirmer lorigine gauloise de cette agglomération, déjà entrevue à l'occasion des travaux du Syndicat d'Initiative en 1969 surveillés par J.-L. Odouze (Rothé, 2001).

Complétant ces données, deux diagnostics réalisés en 2010 et 2013 ont mis en évidence la puissance stratigraphique conservée, qui atteint $5 \mathrm{~m}$ (Viscusi et al., 2014). Dans les deux opérations, les sondages ont révélé des constructions antiques en terre, que la mise en place de blindages a permis de fouiller, sur des surfaces toutefois limitées (9 et $12 \mathrm{~m}^{2}$ ). Ces niveaux d'occupation ont été rencontrés à des profondeurs comprises entre 2,8 et $4,8 \mathrm{~m}$.

Un projet collectif de recherche portant sur les origines de l'agglomération antique jusqu'au haut Moyen Âge a vu le jour en $2015^{\mathbf{1}}$. En reprenant l'intégralité des données et du mobilier issus des découvertes anciennes ou récentes réalisées à Lons-le-Saunier, il doit permettre d'avoir une vision renouvelée de la ville antique.

\section{Une vision fragmentaire mais des vestiges spectaculaires}

Lors du sondage mené rue de la Chevalerie en $2010^{2}$, des constructions en terre, détruites par incendie, ont été mises au jour dans quatre des cinq niveaux d'occupation qui se sont succédé du $\mathrm{I}^{\mathrm{er}}$ au III ${ }^{\mathrm{e}}$ siècle (Viscusi, 2015).

Si une première implantation durant La Tène finale (LTD2) est attestée par la présence d'un sol construit sur les alluvions fluvio-glaciaires, aucune trace d'élévation n'a été observée. En revanche, le sol qui lui succède, mis en place sur un remblai de graviers, était limité par des murs en terre dont seule témoigne la couche de démolition qui le surmonte, provoquée par incendie au cours de la seconde moitié du I ${ }^{\text {er }}$ siècle (état 2).

Les états suivant ont livré des vestiges beaucoup plus explicites. Au cours de la première moitié du II ${ }^{\mathrm{e}}$ siècle (état 3), est édifiée une habitation dont trois pièces ont été partiellement reconnues, séparées par deux murs implantés perpendiculairement [ill. 1]. À l'ouest, la pièce comporte un sol en terre battue surmonté par des lambourdes et un plancher [ill. 2 et 7]. La couche de démolition montre que cet espace était couvert en tuiles et ne comportait pas d'étage ni de plafond. À l'est, la pièce est agrémentée par un sol damé constitué de graviers noyés dans une matrice limoneuse ocre. Dans un second temps, un nouveau sol de composition similaire est aménagé sur un radier de galets. Aucune tuile n'a été recueillie dans cet eśpace probablement pourvu d'une couverture végétale. De la troisième pièce, ne sont connus que deux sols successifs en terre battue. Les murs sont édifiés en terre et montrent que la phase de réfection observée sur les sols s'est étendue à la totalité de l'édifice. Le premier mur, arasé, est monté sur le sol en graviers et limon ocre 


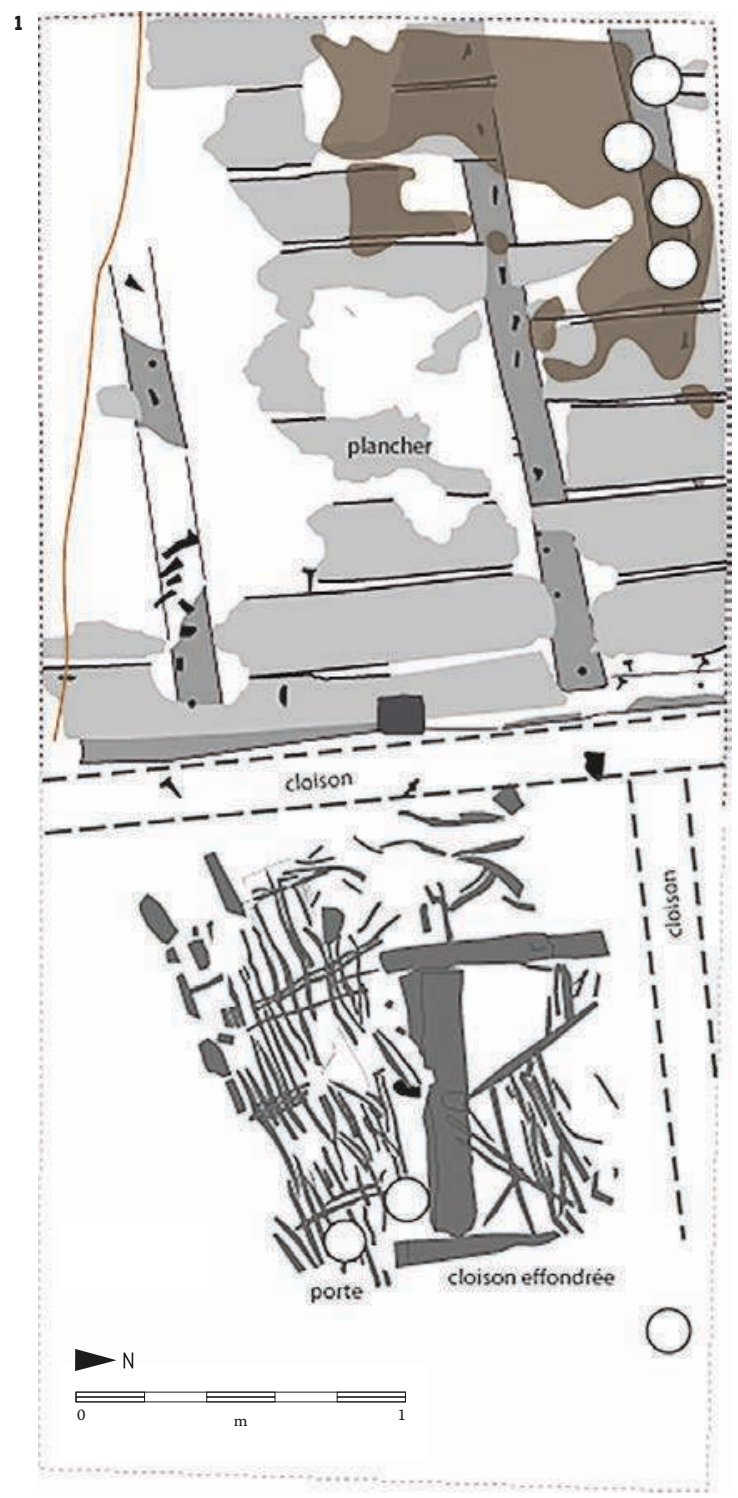

restituer un bâti composé 3. La paroi à pan de bois entrecroisées. Unélément

de trois pièces. et clayonnage effondrée ferreux trouvé à mi-

2. La paroi nord-sud arasée, appartenant à l'état 3 de hauteur de la porte, le long

fouillée rue de la Chevalerie, la rue de la Chevalerie était du montant de la paroi,

s'est affaissée sur le dotée d'une porte dont évoque le système de

plancher de la pièce ouest les longues baguettes fermeture.

durant l'occupation de verticales, majoritairement
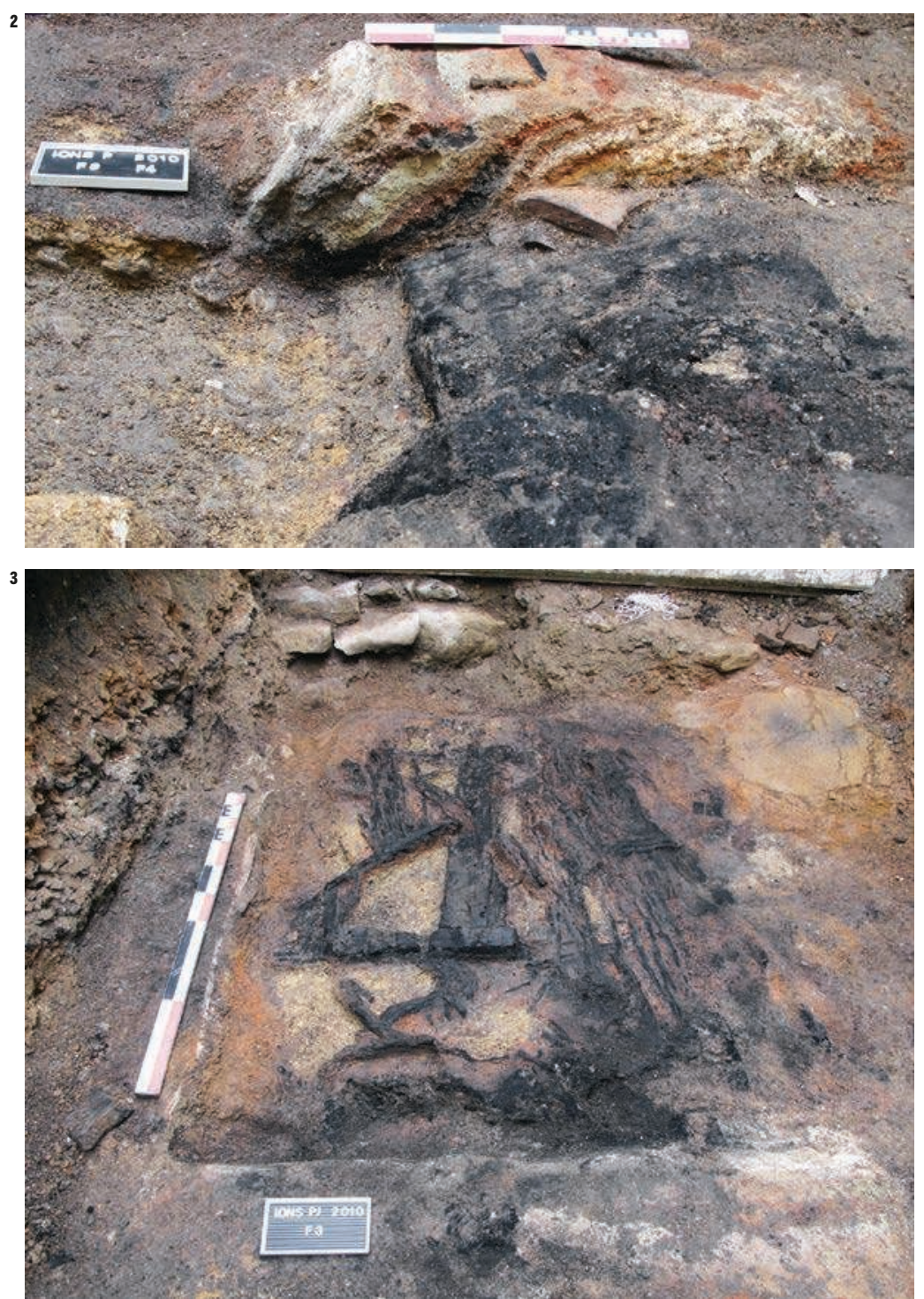


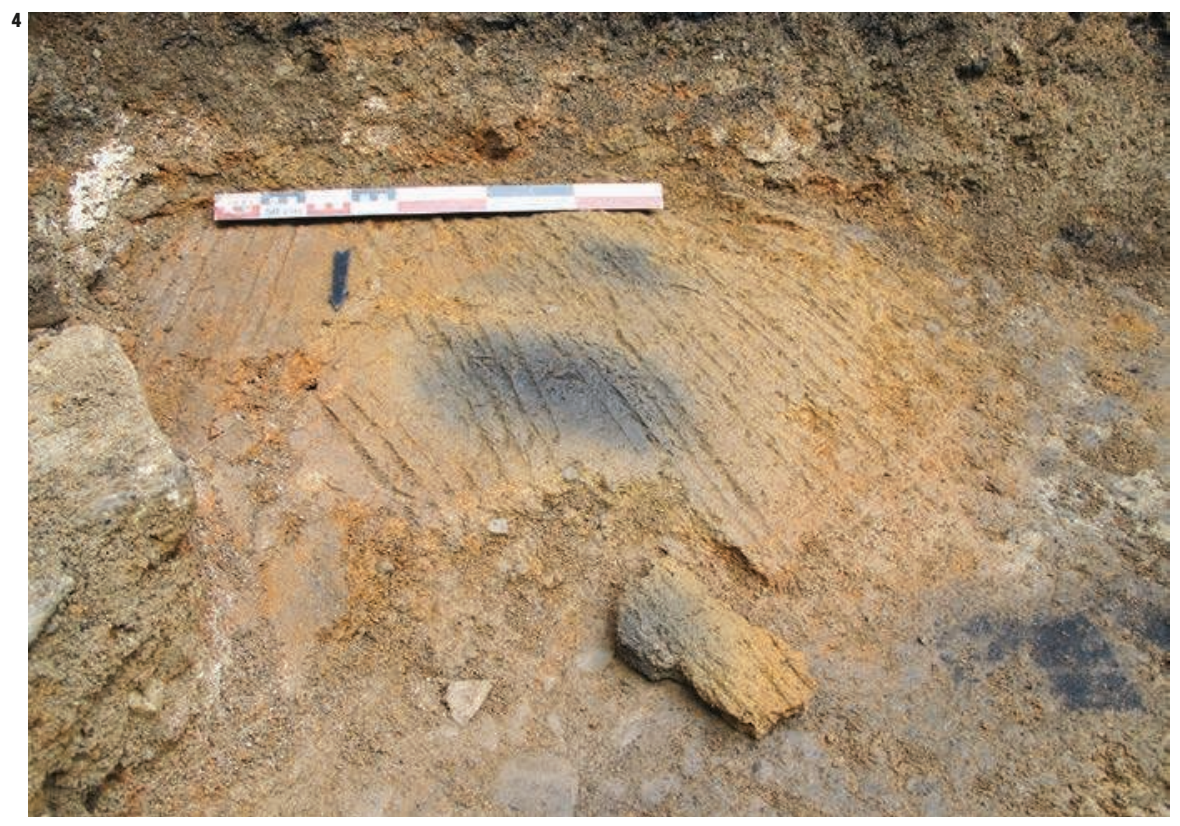

4. Rue de la Chevalerie,

un fragment de paroi cuit

attribué à l'état 3 a révélé

les traces du clayonnage.

5. La partie située au nord

du poteau équarride la

paroi nord-sud de l'état 3

de la rue de la Chevalerie

a subi une combustion

réductrice.

6. Les conditions

d'incendie ont permis

de révéler la technique

de construction de la paro

nord-sud de l'état 3 ,

rue de la Chevalerie.

0
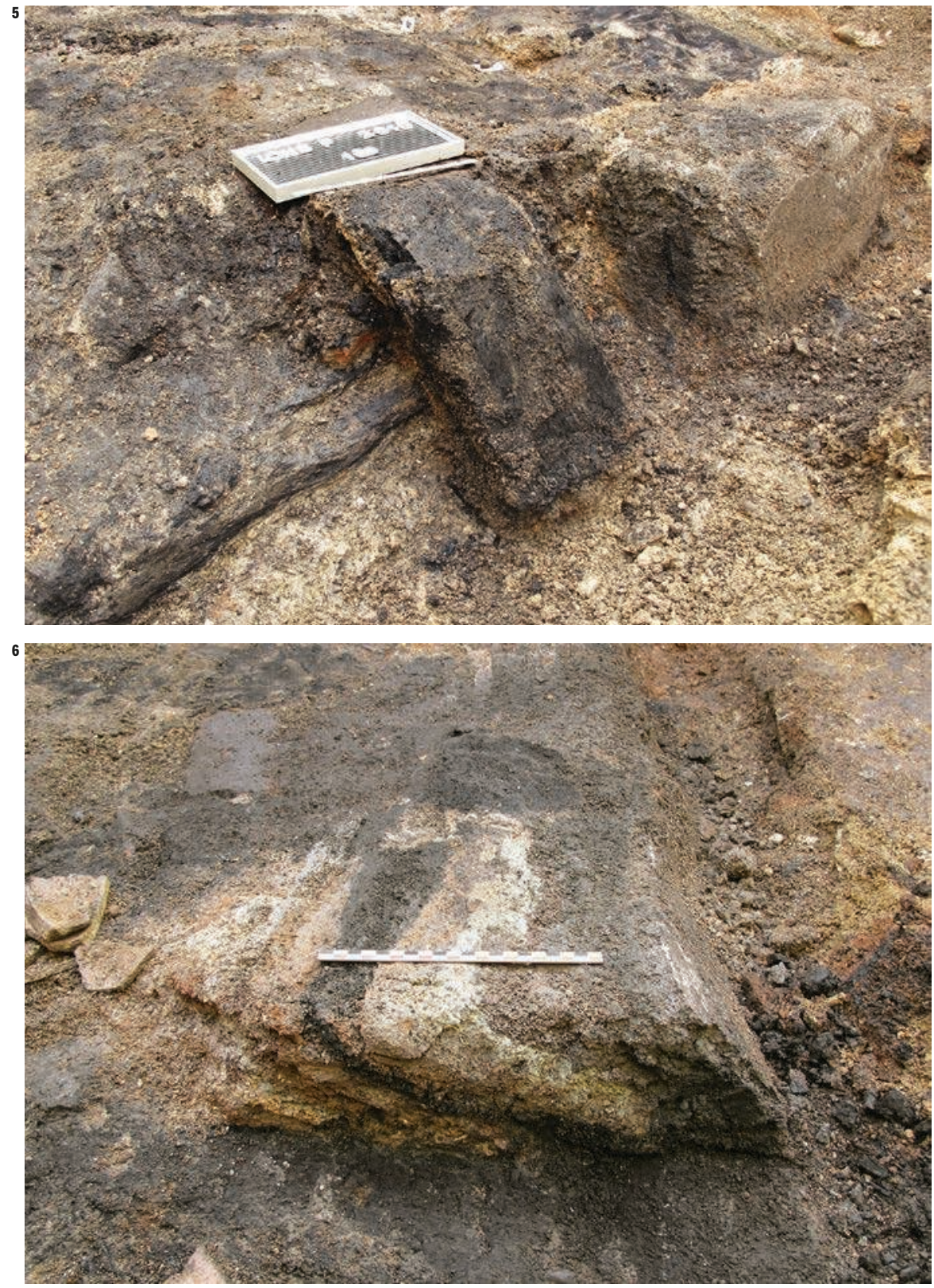
[ill. 2]. Le second semble bâti sur une sablière qui reposait sur le radier de galets et contre laquelle est venu buter le deuxième sol de graviers. Cette paroi à pan de bois s'est effondrée à plat dans la pièce orientale durant un épisode d'incendie [ill. 3].

Les gravats consécutifs à l'incendie ont été régalés sur place, produisant un rehaussement des niveaux d'une trentaine de centimètres, pour permettre la construction d'un nouvel édifice au cours de la seconde moitié du $\mathrm{II}^{\mathrm{e}}$ siècle (état 4). Le nouveau mur ne se superpose pas à ceux des états précédents et son orientation diverge légèrement. La nouvelle construction comporte une fondation en pierre, édifiée dans une tranchée qui incise les remblais de démolition sous-jacents. Le sol contemporain est un plancher, très mal conservé, dont les lambourdes reposent directement sur le toit des remblais. Le sol était couvert par une couche de petits cubes de terre cuite qui constituent les éclats thermiques produits par les tuiles durant l'incendie.

Si le dernier état de construction antique (état 5), occupé au cours du $\mathrm{II}^{\mathrm{e}}$ siècle, a également livré un plancher carbonisé, les gravats produits par l'incendie évoquent une élévation en pierre et mortier de chaux avec couverture en tuiles.

À $150 \mathrm{~m}$ de ces découvertes, des sondages réalisés promenade de la Chevalerie, en $2013^{3}$ (Viscusi Simonin, 2013), ont permis la fouille partielle d'un habitat implanté au début du $\mathrm{I}^{\mathrm{er}}$ siècle de notre ère et détruit par un incendie vers le milieu de ce siècle. La construction est implantée sur plusieurs couches de remblais graveleux préalablement apportés afin de mettre hors d'eau une plateforme située dans le lit majeur de la rivière. La surface observée est divisée en deux pièces par une cloison partiellement conservée en élévation, sur une quinzaine de centimètres, et partiellement effondrée à plat [cf. ill. 8 infra]. Dans la pièce nord, un sol formé d'une calade de galets surmontée par un lit de mortier de chaux prend la suite d'une simple terre battue. La pièce sud ne comporte que des sols successifs en terre battue. L'absence de fragments de tuile et la présence, entre le sol et la paroi effondrée, d'une couche cendreuse et charbonneuse dépourvue de gros charbons évoquent la combustion d'une couverture végétale légère.

\section{L'aspect des vestiges conservés}

Quelques observations s'imposent comme un préalable obligé à l'évocation des techniques de construction. Les maçonneries de terre ont été découvertes sous plusieurs formes : en élévation, effondrées à plat sur le sol, en morceaux de taille décimétrique ou désagrégées dans les remblais de démolition. Si leur destruction est, dans chaque cas, intervenue lors d'un épisode d'incendie, l'impact du feu a été très variable. Ainsi, la température et la présence ou non d'oxygène ont considérablement influencé la texture et la couleur du matériau. Dans un cas, l'action du feu a révélé de façon spectaculaire la composition de la paroi qui n'était pas perceptible de visu à une cinquantaine de centimètres de distance (voir infra).

Sur la plus grande partie de la surface, la paroi à pan de bois effondrée à plat sur le sol de l'état 3 de la rue de la Chevalerie montre une structure en bois équarri et baguettes carbonisées englobées dans une terre de consistance meuble et de couleur jaune. À proximité immédiate, d'autres fragments de parois, découverts superposés sur le même sol, sont totalement cuits [ill. 3 , en haut à droite] et les emplacements des baguettes, entièrement consumées, sont visibles [ill. 4].

Appartenant au même état 3 de la rue de la Chevalerie, une des parois est conservée en élévation sur une hauteur de $40 \mathrm{~cm}$, légèrement affaissée contre la lambourde du plancher voisin [cf. ill. 2 supra]. Sur la faible longueur observée, elle présente une grande variété de coloration. En partie nord, les charbons noirs de la structure interne se distinguent nettement au sein d'une matrice de texture granuleuse qui semble, selon les endroits, soit uniforme, soit formée de deux couches de couleur gris foncé et gris clair [ill. 5]. Au sud, la partie charbonneuse apparaît enrobée par une succession de fines couches de textures et de couleurs variées (blanc, rouge, rose, jaune, noir). Alors que dans le premier cas (au nord), nous sommes tentés d'imaginer l'application d'un torchis en couche unique ou en deux applications englobant le clayonnage et protégé in fine par un enduit, le second cas (au sud) met en évidence la complexité du processus. Il est probable que la technique de construction de la paroi ne varie pas sur la longueur observée mais que seule la variation des conditions de température et d'oxygénation durant l'incendie a permis d'en révéler les étapes.

\section{Des techniques de construction diversifiées}

Les vestiges illustrent la diversité des procédés de construction employés, parfois au sein d'un même édifice. L'emploi simultané de murs de terre et de maçonneries en pierre paraît également avoir existé, comme en témoignent certains indices : durant l'état 3, rue de la Chevalerie, la pièce occidentale, à couverture de tuiles, a produit un remblai de démolition composé d'une grande quantité de chaux alors que la pièce orientale, à couverture végétale, en est dépourvue. Il est donc possible qu'une partie des maçonneries (situées en dehors de l'espace exploré par le sondage) ait été construite en pierre et coexiste avec la paroi qui sépare les deux pièces, édifiée en terre.

Rue de la Chevalerie, le mur qui sépare les deux pièces de l'état 3 comporte un poteau de sapin équarri de $15 \mathrm{~cm}$ de section posé sur le sol [ill. 5]. La paroi en torchis est constituée de fines couches de terre de composition variée, appliquées successivement autour d'une ossature composée de baguettes verticales ou de planches [ill. 6]. Elle est protégée par un enduit couvert d'un lait de chaux. 

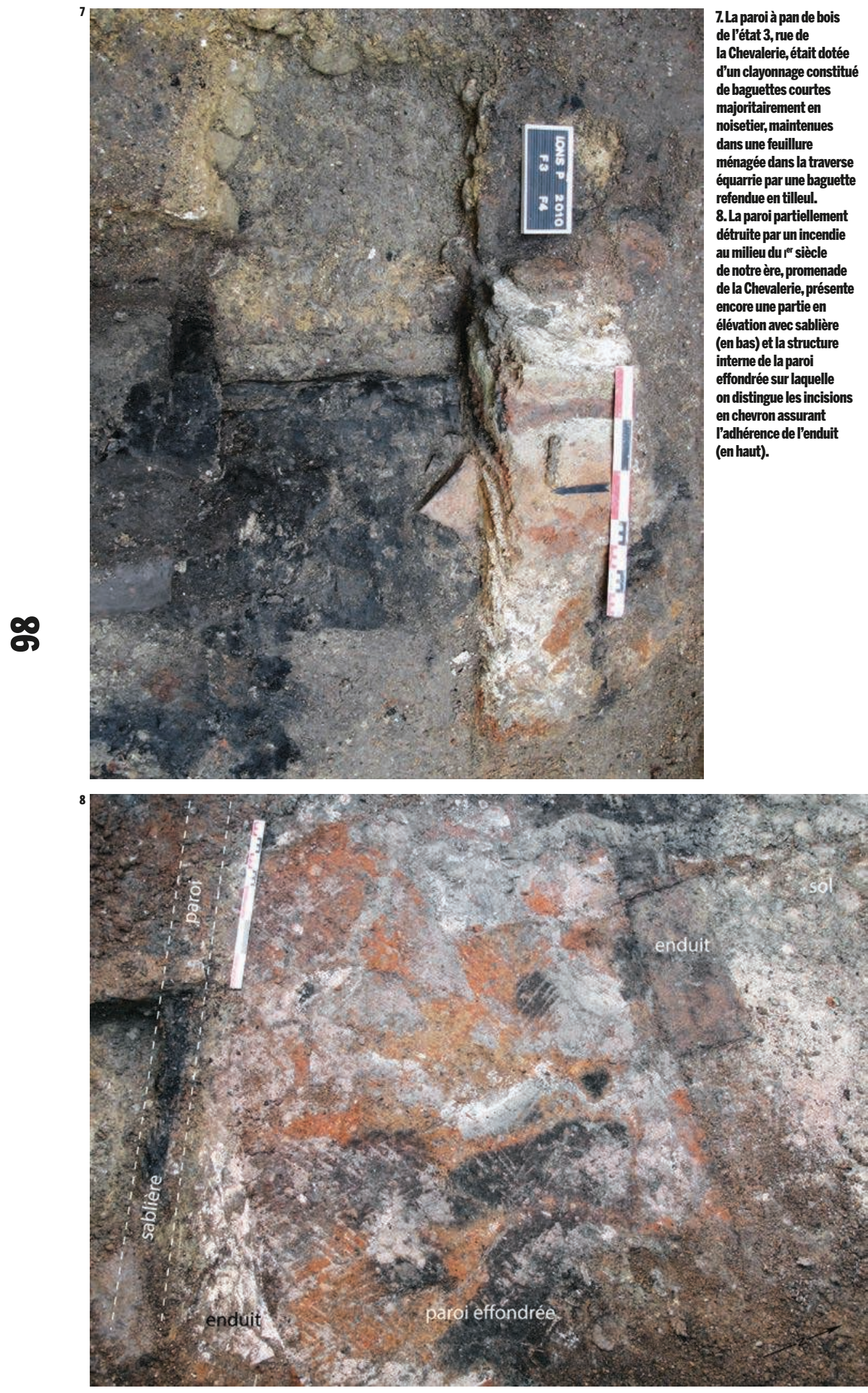

\section{Références bibliographiques}

Baudoux J., aVeC La Participation de Cantrelle S., 2006, « Les habitats gallo-romains en terre et en bois de la rue de la Mésange à Strasbourg », Revue archéologique de l'Est, t. 55, p. 67-102.

Mordefroid J.-L., Cohen A.-S. DE, Mercier C., 1990, Lons-le-Saunier, 2000 ans déjà : la Comédie, fouilles 1989, catalogue d'exposition, Musée d'archéologie de Lons-le-Saunier, Lons-le-Saunier, Cercle Girardot, 33 p. Odouze J.-L., 1985, « La Séquanie », in LASFargues J.

(DIR.), Architectures de terre et de bois, l'habitat privé des provinces occidentales du monde romain, antécédents et prolongements, Protohistoire, Moyen Âge et quelques expériences contemporaines, Paris, éd. de la Maison des Sciences de l'Homme, « Documents d'Archéologie Française », n² 2, p. 85-92.

Paunier D., 1985, « La Suisse », in Lasfargues J. (Dir.), Architectures de terre et de bois, l'habitat prive des provinces occidentales du monde romain, antécédents et prolongements, Protohistoire, Moyen Âge et quelques expériences contemporaines, Paris, éd. de la Maison des Sciences del'Homme, «Documents d'Archéologie Française », n' ${ }^{\circ}$ 2, p. 113-126.

Rothe M.-P., 2001, «Lons-le-Saunier », dans Le Jura. Carte archéologique de la Gaule, 39, Pré-inventaire archéologique publié sous la dir. de M. Provost, Paris, Académie des Inscriptions et Belles-Lettres, p. 453-471. VIscusi Simonin V. (DIR.), 2013, Franche-Comté, Jura Lons-le-Saunier, Promenade de la Chevalerie, De La Tène finale à nos jours, $5 \mathrm{~m}$ de stratigraphie aux marges de la ville antique et médiévale, rapport d'opération, SRA Franche-Comté, 274 p., 164 fig. Viscusi V., JACCOTTEy L. et HumBert S., 2014, « Habitat antique et sépultures du haut Moyen Âge mis au jour à Lons-le-Saunier », Bulletin de la Société d'émulation du Jura, travaux 2013, p. 93-116. cnum.cnam.fr/CGI/redir. cgi?FOLRESQE2 (consulté le 29/o3/2015). VISCUSI V. (DIR.), 2015, Franche-Comté, Jura, Lons-leSaunier, rue de la Chevalerie, 5,5 $\mathrm{m}$ de stratigraphie de La Tène finale à nos jours, cinq épisodes dincendie, un cimetière du haut Moyen Âge, rapport de diagnostic, Inrap/SRA Franche-Comté, 164 p., 127 fig. 
Ces deux dispositifs ont été observés à Villardsd'Héria dans le Jura (Odouze, 1985), où les baguettes verticales sont associées à une sablière, et à Strasbourg dans le Haut-Rhin (Baudoux, 2006) ou Augst en Suisse (Paunier, 1985) où certaines parois montrent une structure interne en planches.

La paroi effondrée à plat sur le sol de la pièce voisine du mur précédent renferme une structure à pan de bois et clayonnage. Un montant et deux traverses carbonisés sont parfaitement conservés [cf. ill. 3 supra] tandis que quelques charbons pourraient évoquer une sablière. La structure interne est formée par des baguettes maintenues dans la traverse par une baguette d'assemblage refendue disposée dans une feuillure [ill. 7]. Les essences d'arbre utilisées sont très variées (sapin et hêtre pour les montants et traverses, noisetier, hêtre et érable principalement pour les baguettes) ${ }^{\mathbf{4}}$. La terre qui englobe la structure montre une texture fine

et homogène et la surface ne comporte pas d'enduit.

La paroi découverte promenade de la Chevalerie, large d'une vingtaine de centimètres, a été édifiée sur une sablière en chêne. Aucune autre trace de structure interne n'a été détectée, tant dans la quinzaine de centimètres conservée en élévation que dans les parties effondrées à plat. Celles-ci sont constituées de couches de textures différentes, limoneuses, argileuses ou sableuses, et de couleurs différentes, orangées ou grises. Des incisions disposées en chevrons ont été pratiquées dans la surface terreuse pour une meilleure adhérence du mortier sableux recouvert d'un fin enduit de chaux blanc [ill. 8]. Certains autres fragments semblent couverts par un enduit terreux et sableux dépourvu de finition à la chaux.

Si les vestiges de l'état 4 de la rue de la Chevalerie ne conservent qu'une fondation en pierre dépourvue de liant, plusieurs indices évoquent une élévation en terre sur solin maçonné. La couche de démolition produite par l'incendie des élévations était composée de fragments de parois carbonisés. La coupe a montré la présence d'une tranchée d'épierrement qui contenait du mortier de chaux et des pierres de petites dimensions.

Létude des techniques de construction n'est pas achevée. L'observation détaillée de la terre des parois ${ }^{5}$ et celle des dégraissants végétaux ${ }^{6}$ sont prévues dans le cadre du PCR portant sur l'agglomération antique de Lons-le-Saunier.

\section{La fonction et le statut des constructions}

Les pièces de l'état 3 de la rue de la Chevalerie ont livré des vestiges permettant de caractériser leur occupation. Sur le plancher de la pièce occidentale, à couverture de tuiles, ont été recueillis plusieurs pots de stockage brisés sur place, qui avaient libéré leur contenu sous la forme d'une nappe de graines carbonisées. La pièce orientale, à couverture végétale, n’a livré que quelques fragments de pots. Durant l'état suivant (état 4), la pièce pourvue d'un plancher abritait également des réserves de graines, découvertes sous la forme d'amas localisés qui trahissent leur conservation dans des contenants périssables. Sous la promenade de la Chevalerie, un foyer était encastré dans le sol en terre battue du premier état.

Si ces vestiges trahissent une activité domestique, rien ne permet toutefois d'affirmer que nous sommes ici en présence de la pièce à vivre d'un habitat modeste ou d'une pièce spécialisée dans une construction plus vaste à fonctions diversifiées.

Le choix de la terre à construire est, à Lonsle-Saunier, indépendant du milieu naturel. En effet, l'environnement géologique proche (rebord du plateau jurassien) constitue une source d'approvisionnement en pierre abondante et disponible immédiatement.

Dans les fenêtres de surface très restreinte ouvertes sur l'occupation antique de l'agglomération lédonienne à l'occasion de deux opérations de sondages, la conservation et létude des élévations en terre ont été permises par les incendies qui ont affecté la totalité des niveaux d'occupation. Les constructions mises au jour peuvent être assimilées à des habitats modestes, peut-être regroupés au sein d'un quartier. Alors que, pour les même périodes, la fouille de la place de la Comédie avait livré des bâtiments avec enduits peints et traces de mosaïque, les demeures entrevues dans les sondages sont dépourvues d'étage et comportent des murs en terre qui ne semblent pas toujours protégés par un enduit de chaux et des sols en terre battue ou des planchers.

Les modes constructifs employés montrent une évolution entre le $\mathrm{I}^{\mathrm{er}}$ siècle de notre ère et le $\mathrm{III}^{\mathrm{e}}$ siècle. Les premières constructions font un usage exclusif de la terre, tant pour les sols que pour les élévations, qui supportent des couvertures légères. $\mathrm{Au} \mathrm{II}{ }^{\mathrm{e}}$ siècle, les bâtiments semblent comporter aussi bien des élévations en terre qu'en pierre ou des élévations mixtes, à fondation et solin de pierre et élévation en terre. La terre est mise en œuvre sous la forme de torchis, parfois appliqué en fines couches de compositions différentes. Les planchers coexistent avec des sols de terre et graviers et la toiture tuilée côtoie la couverture végétale. En revanche, le niveau d'occupation du III $^{\mathrm{e}}$ siècle n'a livré les traces que d'une construction maçonnée en pierre au sol planchéié.
4. Détermination Olivie Girardclos, laboratoir Chrono-environnement, UMR 6249, et Étienne Chabrol (dans le cadre d'un Master 1).

5. Delphine Minni, Inrap 6. Emmanuelle Bonnaire Inrap. 